\title{
Semi-automated Approach to Hippocampus Segmentation Using Snake from Brain MRI
}

\author{
Ala'a ddin Al Shidaifat ${ }^{\dagger}$, Ramadan Al-Shdefat ${ }^{\dagger+}$, Heung-Kook Choi $^{++\dagger}$
}

\begin{abstract}
The hippocampus has been known as one of the most important structure related to many neurological disorders, such as Alzheimer's disease. This paper presents the snake model to segment hippocampus from brain MRI. The snake model or active contour model is widely used in medical image processing fields, especially image segmentation they look onto nearby edge, localizing them accurately. We applied a snake model on brain MRI. Then we compared our results with an active shape approach. The results show that hippocampus was successfully segmented by the snake model.
\end{abstract}

Key words: Hippocampus, Snake model, Segmentation, MRI, Active shape approach

\section{INTRODUCTION}

The development of medical imaging technologies in last three decades has grown and enormously increased its important in the diagnosis of diseases [1]. The original snake model was introduced by Kass et al. [2], which defines a curve within an image evolving through internal force from the inside of the curve and external force computed from the image data and segmenting the targeted object.

The hippocampus is an important structure of the human limbic system, which plays an essential role in learning and memory processing. In particular, researchers have shown that abnormalities in the volume and architecture of the hippocampus are associated with a number of neurological and psychiatric illnesses, including mild cognitive impairment (MCI) [3-4], Alzheimer's disease [5-7],

※ Corresponding Author: Heung-Kook Choi, Address: (621-749) 197 Injero, Department of Computer Engineering, UHRC Inje University, Gim-Hae, Korea, TEL : +82-55 320-3437, FAX: +82-55 322-3107, E-mail : cschk@inje.ac.kr

Receipt date: Feb. 9, 2014, Revision date: Mar. 18, 2014 Approval date: Apr. 22, 2014

+ Department of Computer Engineering, UHRC, Inje University, Korea (E-mail : alaasoftware@hotmail.com) epilepsy [8], and schizophrenia [9-11].

Since the early 1990's, MRI has been used to produce accurate hippocampal volume measurements by separating hippocampal structures not only from the surrounding white matter (WM), but also from contiguous areas of gray matter (GM) [11-12]

The hippocampus is a small GM structure that is adjacent to other GM structures e.g., amygdala, parahippocampal gyrus [13]. This specialty of the hippocampus means that in brain MRI, the hippocampus has relatively low contrast and no distinguishable boundaries along significant portions of its surface [12]. Fig. 1 shows the position and intensity of hippocampus in sagittal MRI.

To this date, three different approaches have been proposed for hippocampus segmentation: manual, fully automatic, and semi-automatic segmentation methods.

\footnotetext{
${ }^{++}$Dept. of Pharmaceutics, Salman Bin Abdulaziz University, Saudi Arabia (E-mail : rshdefat@yahoo.com)

${ }^{+++}$Department of Computer Engineering, UHRC, Inje University, Korea

※ This research was supported by Basic Science Research Program through the National Research Foundation of Korea (NRF) funded by the Ministry of Education, Science and Technology (2012-0002646).
} 


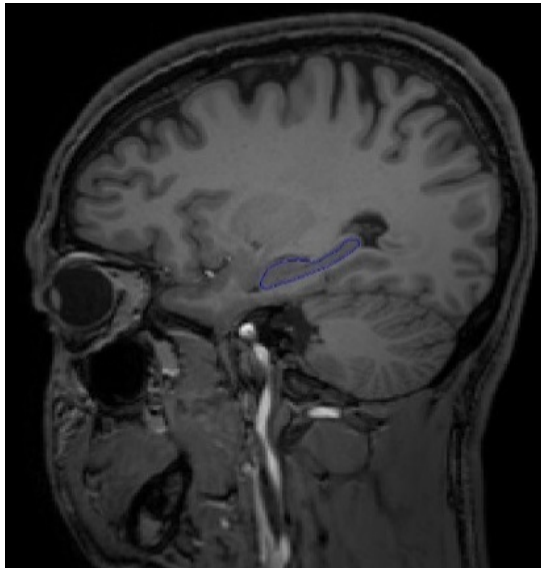

Fig. 1. Position and intensity of hippocampus in sagittal MRI.

In manual segmentation, an expert rater identifies and labels the hippocampus on each slice of brain MRI set. This method requires extensive human interaction and considerable training, and intra-rater reliability may be difficult to achieve.

A fully automatic method is ideal, but the segmentation of the hippocampi with conventional methods, such as edge tracking, thresholding, or region growing, is not reliable, due to the small size, low contrast, and apparent discontinuity of the edges of the hippocampus. Webb et al. [14] proposed for automatic segmentation involves warping of an atlas to the individual brain MRI. However, when the internal object is relatively small and its shape is highly variable, as it is the case for the hippocampus, this approach may not generate an accurate result, due to its sensitivity to the imperfections involved with the registration and warping steps.

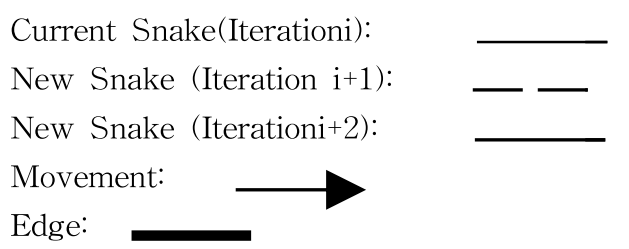

Semi-automatic methods may provide a more realistic approach for hippocampus segmentation because they combine the automatic techniques with a priori knowledge of the hippocampal location, anatomical boundaries and shape [13]. We apply a semi-automatic snake model for the accurate hippocampus segmentation.

The remainder of this paper is organized as follows. We explain the snake model in section 2 . Section 3 provides implementation details and compares results of the snake model and the active shape approach. In section 4, we state our conclusion.

\section{SNAKE MODEL}

A snake is a parametric contour that deforms over a series of iterations by time steps. Therefore each element $x$ along the contour depends on two parameters:

$$
x=x(s, t)
$$

where $s$ is space (curve) parameter and usually varies between 0 and $1, t$ is time (iteration) parameter.

The total energy of the model $E_{\text {snake }}$ is given by the sum of the energy for the individual snake elements:

$$
E_{\text {snake }}=\int_{0}^{1} E_{\text {element }}(x(s)) d s
$$

The integral notation used in equation(1) implies an open-ended snake; however, joining the first and last elements makes the snake into a closed loop as shown in Fig. 2.

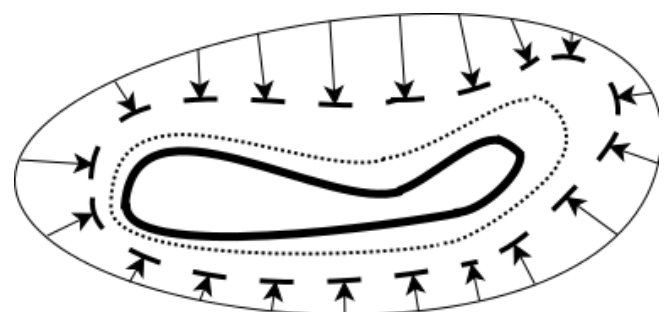

Fig. 2. A closed active contour model. 
Over a series of time steps the snake moves into alignment with the nearest edge. The contour is influenced by internal and external constraints, and by image forces. Internal constraints give the model tension and stiffness. External constraints come from initialization procedures. Image energy is used to drive the model towards edges. Equation (1) can be rewritten in terms of three basic energy functions:

$$
E_{\text {snake }}=\int_{0}^{1} E_{\text {internal }}(x) d s+\int_{0}^{1} E_{\text {external }}(x) d s+\int_{0}^{1} E_{\text {image }}(x) d s
$$

The internal, external energy functions and image energy are discussed next.

\subsection{Internal Energy}

The following equation describes the internal energy of a snake element:

$$
\begin{aligned}
& E_{\text {internal }}=E_{\text {Tension }}+E_{\text {Siffiness }} \\
& E_{\text {Tension }}=\alpha(s)\left|x_{s}(s)\right|^{2} \\
& E_{\text {Stiffiess }}=\beta(s)\left|x_{s s}(s)\right|^{2}
\end{aligned}
$$

This energy contains a first-order term controlled by $\alpha(s)$, and a second-order term controlled by $\beta(\mathrm{s})$. The first-order term makes the snake act like a rubber band representing tension; the sec $^{-}$ ond-order term makes it resist bending by producing stiffness in [15] and [16].

If the other terms were not applied the contour will keep shrinking to a single point. The changing weights $\alpha(s)$ and $\beta(\mathrm{s})$ control the relative importance of the tension and stiffness terms. If we set $\beta(\mathrm{s})$ to zero meaning that the second order is continuous will the model to have a corner.

\subsection{External Energy}

The external energy of snake model discussed by $\mathrm{Xu}$ and Prince in [17], it is responsible for putting the snake near the desired local minimum [2], the initialization procedures are used to manage both of the attraction and repulsion forces which control the active contour models to or from the desired features. The external energy term representing the attraction is:

$$
E_{\text {external }}(x)=k|i-x|^{2}
$$

An attraction force similar to spring force is generated between a snake element and a point $i$ in an image. This energy is minimal when $x=i$, and it takes the value of $k$ when $i-x= \pm 1$. The external energy function which represents the repulsion show in next equation:

$$
E_{\text {external }}(x)=\frac{k}{|i-x|^{2}}
$$

This repulsion energy we can say it is like a volcano pushing out of one local minimum into another, when $x=i$ we get maximum energy. The $\mathrm{re}^{-}$ pulsion term must be stopped as $i-x \rightarrow 0$.

\subsection{Image (Potential) Energy}

There are three different potential energies $P$ produced by the processing of the image $I(x, y) \mathrm{re}^{-}$ sults a force that is used to control snakes towards the features of interest. These energy attract snakes to lines, edges and terminations shown in (2), (3) and (4) respectively. The total potential energy can be expressed as a weighted combination of these three functions:

$$
\begin{aligned}
P & =E_{\text {inage }}=w_{\text {line }} E_{\text {line }}+w_{\text {edge }} E_{\text {edge }}+w_{\text {term }} E_{\text {term }} \\
E_{\text {line }} & =\int_{0}^{1} I(x(s)) d s \\
E_{\text {edge }} & =-|\nabla I(x(s))|^{2} \\
E_{\text {term }} & =\int_{0}^{1} \frac{\partial \theta}{\partial n_{\perp}} d s=\int_{0}^{1} \frac{\partial^{2} C / \partial n^{2} \perp}{\partial C / \partial n} d s \\
E_{\text {term }} & =\int_{0}^{1} \frac{C_{y y} C_{x}^{2}+C_{x x} C_{y}^{2}-2 C_{x y} C_{x} C_{y}}{\left(C_{x}^{2}+C_{y}^{2}\right)^{3 / 2}} d s
\end{aligned}
$$

\section{EXPERIMENTAL RESULTS}

The brain images were acquired using an Achieva Philips 3.0T MR imaging scanner from 
Haeundae Paik Hospital in Busan, Korea. The subjects were 27 years old normal male.

In Fig. 3, the first column shows the test images, which area T1-weighted grayscale DICOM image with a $256 * 256$ resolution. The second column shows the results of hippocampus segmentation using the snake model. The initial points are placed around the hippocampus boundary manually by the user and the hippocampus can be segmented accurately. The initialization can be placed far from the hippocampus, if there is no fake edges interrupt between initialization and hippocampus boundary. The segmentation result of snake model depends
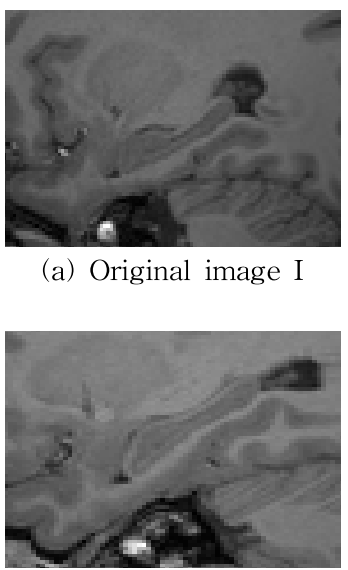

(c) Original image II

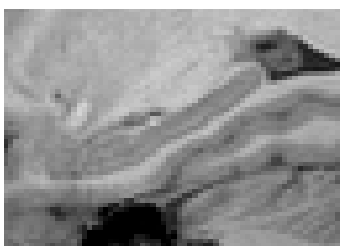

(e) Original image III

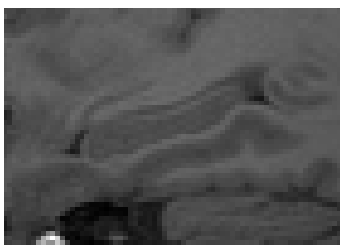

(g) Original image IV (a) Original image I

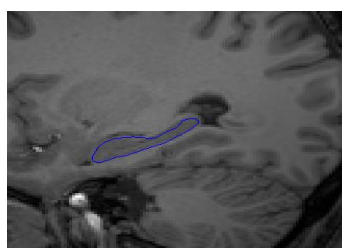

(b) Snake segmentation result

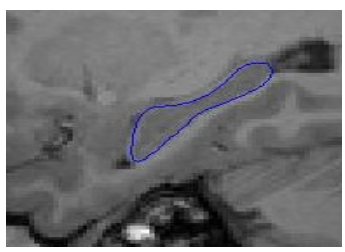

(d) Snake segmentation result

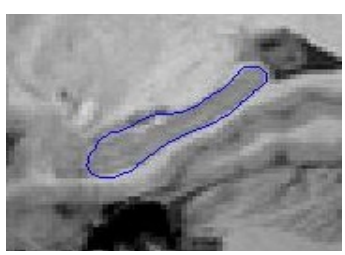

(f) Snake segmentation result

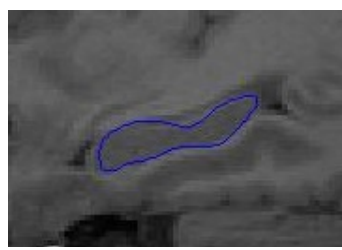

(h) Snake segmentation result
Fig. 3. First column: original images; Second column: the segmentation results of Snake. on several weight energy parameters. In our $\mathrm{ex}^{-}$ periment we set them as follows:

- The tension controls the first derivative term of contour, $\alpha(s)=10^{-4}$

- The stiffness controls the second derivative term of contour, $\beta(\mathrm{s})=0.2$

- The weighting factor for intensity, edge and terminate based on potential terms, $w_{\text {line }}=0$, $w_{\text {edge }}=5, w_{\text {term }}=0$

The number of iterations were controlled the segmentation accuracy.

In the Fig. 4, the first column shows the ground truth for validation of segmentation results, hippocampus regions of subjects were manually drawn by ITKSNAP 1.6 [18]. The second column shows the binary images of snake model results and the third column shows the binary images results of the active shape approach segmentation [19].

The percentage accuracy evaluation of snake model segmentation is acquired by using Jaccard coefficient measurement in [20]. Jaccard coefficient can be computed based on the number of elements in the intersection set divided by the number of elements in the union set. The segmentation is matched to the manually provided ground truth according to the equation (5).

$$
P(A, B)=\frac{|A \cap B|}{|A \cup B|} \times 100 \%
$$

where $P(A, B)$ represents the segmentation accuracy in percentage, $A$ and $B$ represent the binary images of ground truth and segmentation result of target hippocampus respectively.

The results show that the snake model works well, the segmentation accuracy is higher and also faster when compared to the active shape approach as it is shown in the Table 1.

\section{CONCLUSION}

In this paper, we applied the snake model to segment hippocampus from brain MR images, which 


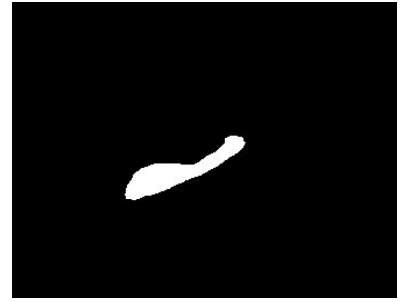

(a) Ground truth image I

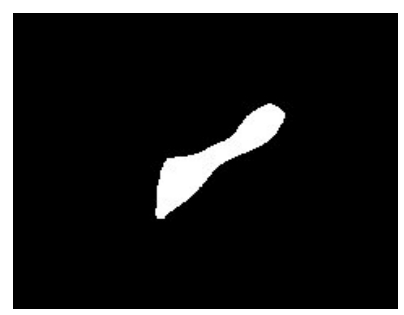

(d) Ground truth image II

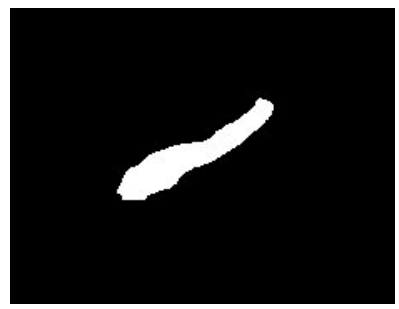

(g) Ground truth image III

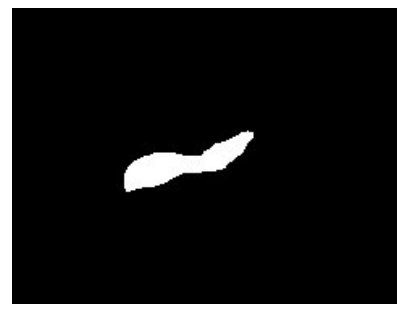

(j) Ground truth image IV

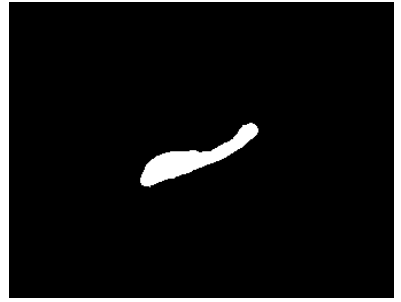

(b) Snake segmentation result

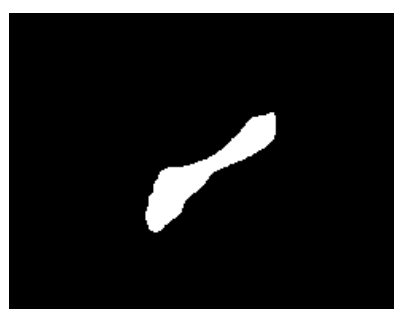

(e) Snake segmentation result

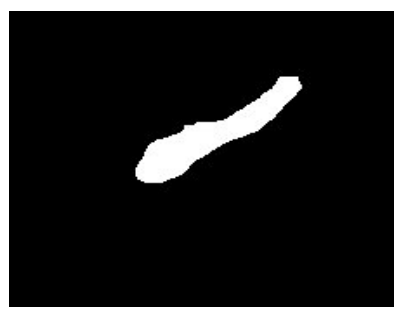

(h) Snake segmentation result

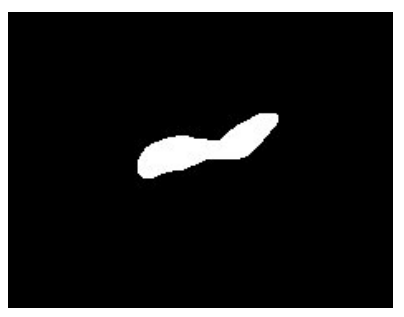

(k) Snake segmentation result

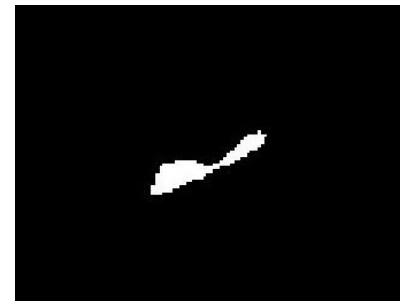

(c) Active shape segmentation result

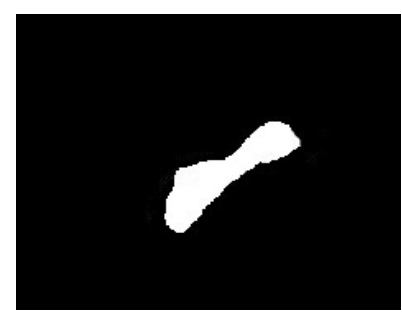

(f) Active shape segmentation result

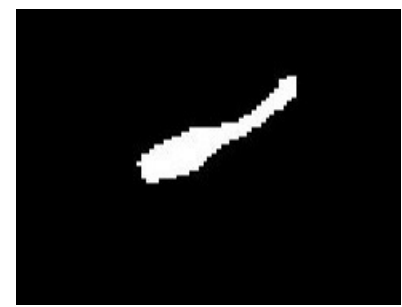

(i) Active shape segmentation result

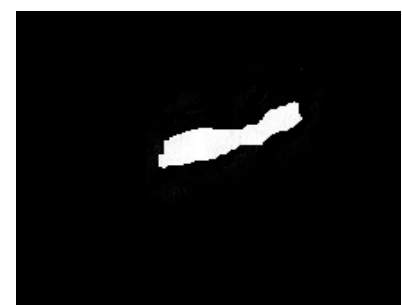

(1) Active shape segmentation result

Fig. 4. The first column: the ground truth of the hippocampus, Second column: result of snake model, third column: the result of active shape approach.

Table 1 The segmentation accuracy and the elapsed time of snake model and active shape approach

\begin{tabular}{|l|c|c|c|c|}
\hline \multirow{2}{*}{} & \multicolumn{2}{|c|}{ Accuracy of segmentation (\%) } & \multicolumn{2}{c|}{ Elapsed time (sec) } \\
\cline { 2 - 5 } & Snake & Active shape & Snake & Active shape \\
\hline \hline Original image I & 91.51 & 71.28 & 19 & 60 \\
\hline Original image II & 85.83 & 79.05 & 26 & 57 \\
\hline Original image III & 85.05 & 74.31 & 24 & 55 \\
\hline Original image IV & 80.02 & 73.49 & 21 & 56 \\
\hline
\end{tabular}

were provided from Haeundae Paik Hospital in Busan, Korea. We compared our segmentation results with the results of active shape approach by referring to the ground truth of the hippocampus. The Jaccard coefficient measurement was used to evaluate segmentation accuracy. Our results were 
more accurate and faster than the active shape model. However, the snake model still needs to be optimized and also the ground truth of the hippocampus should be segmented by the expert.

\section{REFERENCES}

[1] N. Sengee, A. Sengee, A. Enkhbolor, and H. K. Choi, "Contrast Enhancement for Segmentation of Hippocampus on Brain MR Images," Journal of Korea Multimedia Society, Vol. 15, No. 12, pp. 1409-1416, 2012.

[2] M. Kass, A. Witkin, and D. Terzopoulos, "Snakes: Active Contour Models," International Journal of Computer Vision, Vol. 1, No. 4, pp. 321-331, 1988

[3] W.G. Jeon, Y.S. Izmantoko, J.H. Son, and H.K. Choi, "Hippocampus Volume Measurement for the Determination of MCI," Journal of Korea Multimedia Society, Vol. 15, No. 12, pp. 1449-1455, 2012.

[4] H. Hampel, S.J. Teipel, T. Fuchsberger, N. Andreasen, J. Wiltfang, M. Otto, et. al. "Value of CSF Beta-amyloid1-42 and Tau as Predictors of Alzheimer's Disease in Patients with Mild Cognitive Impairment," Molecular Psychiatry, Vol. 9, No. 6, pp. 526-532, 2009.

[5] J.G. Csernansky, L. Wang, S. Joshi, J.P. Miller, M. Gado, D. Kido, et. al. "Early DAT is Distinguished from Aging by High-dimensional Mapping of the Hippocampus," Neurology, Vol. 55, pp. 1636-1643, 2000.

[6] M.J. Firbank, A.M. Blamire, M.S. Krishnan, A. Teodorczuk, P. English, A. Gholkar, et. al. "Atrophy is Associated with Posterior Cingulate White Matter Disruption in Dementia with Lewy bodies and Alzheimer's Disease," NeuroImage, Vol. 36, No. 1, pp. 1-7, 2007.

[7] R. Brookmeyer, E. Johnson, K. Ziegler-Graham, and H.M. Arrighi, "Forecasting the Global Burden of Alzheimer's Disease," Alzheimer's and Dementia, Vol. 3, No. 3, pp. 186-191, 2007.
[8] F. Cendes, Z. Caramanos, F. Andermann, F. Dubeau, and D.L. Arnold, "Proton Magnetic Resonance Spectroscopic Imaging and Magnetic Resonance Imaging Volumetry in the Lateralization of Temporal Lobe Epilepsy: A Series of 100 Patiekts," Annals of Neurology, Vol. 42, No. 5, pp. 737-746, 1997.

[9] H.C. Strasser, J. Lilyestrom, E.R. Ashby, N.A. Honeycutt, D.J. Schretlen, A.E. Pulver, et. al. "Hippocampal and Ventricular Volumes in Psychotic and Nonpsychotic Bipolar Patients Compared with Schizophrenia Patients and Community Control subjects: A Pilot Study," Biological Psychiatry, Vol. 57, No. 6, pp. 633639, 2005.

[10] S.Keihaninejad, R.A. Heckemann, I.S. Gousias, P. Aljabar, J.V. Hajnal, D. Rueckert, et. al. "Automatic Volumetry can Reveal Visually Undetected Disease Features on Brain MR Images in Temporal Lobe Epilepsy," Proceeding of the IEEE International Symposium on Biomedical Imaging, pp. 105-108, 2010.

[11] J.I. Arribas, V.D. Calhoun, and T. Adali, "Automatic Bayesian Classification of Healthy Controls, Bipolar Disorder, and Schizophrenia using Intrinsic Connectivity Maps from fMRI Data," IEEE Transactions on Biomedical Engineering, Vol. 57, No. 12, pp. 2850-2860, 2010.

[12] D. Shen, S. Moffat, S.M. Resnick, and C. Davatzikos, "Measuring Size and Shape of the Hippocampus in MR Images using a Deformable Shape Model," NeuroImage, Vol. 15, No. 2, pp. 422-434, 2002.

[13] X. Lu and S. Luo, "Segmentation of Hippocampus in MRI using Watersnakes," Proceeding of the IEEE International Conference on Complex Medical Engineering, pp. 552555, 2007.

[14] J. Webb, A. Guimond, P. Eldridge, D. Chadwick, J. Meunier, J.P. Thirion, et. al. "Auto- 
matic Detection of Hippocampal Atrophy on Magnetic Resonance Images," Magnetic Resonance Imaging, Vol. 17, pp. 1149-1161, 1999.

[15] B. Ostlad and A. Tonp, "Encoding of a Priori Information in Active Contour Models," IEEE Transactions on Pattern Analysis Machine Intelligence, Vol. 18, No. 9, pp. 863-872, 1996.

[16] S.C. Zhu and A. Yuille, "Region Competition: Unifying Snakes, Region Growing, and Bayes/ MDL for Multiband Image Segmentation," IEEE Transactions on Pattern Analysis Machine Intelligence, Vol. 18, No. 9, pp. 884900, 1996.

[17] C. Xu and J. Prince, "Snakes, Shapes, and Gradient Vector Flow," IEEE Transaction on Image Processing, Vol. 7, No. 3, pp. 359-369, 1998.

[18] ITK-SNAP, http://www.itksnap.org, 2013.

[19] A. Enkhbolor, Y.S. Izmantoko, and H.K. Choi, "Comparison of Active Contour and Active Shape Approaches for Corpus Callosum Segmentation," Journal of Korea Multimedia Society, Vol. 16, No. 9, pp. 1018-1030, 2013.

[20] C. Held, R. Palmisano, L. Haberle, and T. Wittenberg, "Comparison of Parameteradapted Segmentation Methods for Fluorescence Micrographs," Cytometry Part A., Vol. 79, No. 11, pp. 933-945, 2011.

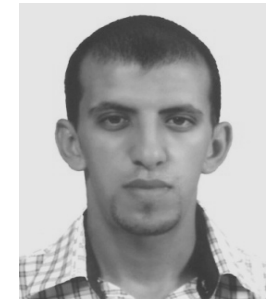

Ala'addin Al Shidaifat

received the B.S. degree in Software Engineering from $\mathrm{Al}^{-}$ Hussein Bin Talal University, Jordan, in 2011. Currently, he is a master student in Computer Engineering department of Inje University, South Korea, joining Medical Image Technology Laboratory (MITL). His research interests are image enhancement, image segmentation, and image visualization.

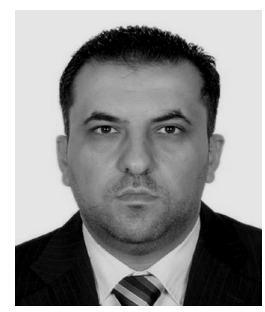

\section{Ramadan I. Al-Shdefat}

is an Assistant Professor of Pharmaceutics, College of Pharmacy, Salman Bin Abdulaziz, Saudi Arabia. He is the director of Research center, College of Pharmacy, Salman bin Abdulaziz University from 2011 until date. He obtained his Ph.D. degree in Pharmaceutical sciences from The State University of Medicine and Pharmacy (Chisinau, Republic of Moldova). He has good experience in development of Novel drug delivery Systems and their in vitro and in vivo analytical and bio-analytical evaluations. He has very good knowledge of scientific and ethical aspects of pre-clinical and clinical research and observed a number of bio- $^{-}$ equivalence studies. He has done significant original work and published many research articles in national and international journals.

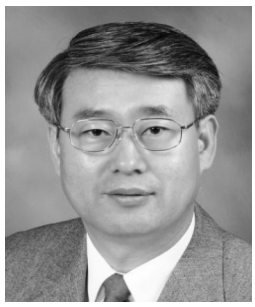

Heung-Kook Choi

has gone the undergraduate studying and graduate studying in computer science and engineering at the Department of Electrical Engineering of Linköping University, Sweden (19841990) and Ph.D. studying in computerized image analysis at the Center for Image Analysis of Uppsala University, Sweden (1990-1996). He was President of Industry and Academic Cooperation Foundation at Inje University and now he is President of Korea Multimedia Society. His interesting research fields are in computer graphics, virtual reality, and medical image processing and analysis. 